\title{
Studying the Progenitors of Type Ia Supernovae via Lensing with the Kepler Survey
}

\author{
Rosanne Di Stefano \\ Harvard-Smithsonian Center for Astrophysics, \\ 60 Garden St., Cambridge MA 02138 \\ email: rd@cfa.harvard.edu
}

\begin{abstract}
Every model for the progenitors of Type Ia supernovae (SNe Ia) requires that binaries pass through an epoch during which a white dwarf (WD) orbits a non-degenerate star. Depending on the mass of the WD, the radius of its companion, and the orbital separation, the WD may lens its companion. The lensing event would be an antitransit, an increase in light from the companion that can rise to the level of a percent or more, during an interval of hours. Antitransits are periodic. By studying them we can determine the properties of both the WD and its companion, as well as the characteristics of the orbit. Lensing events of this type are almost certain to be observed by the Kepler mission, while some can even be detected by groundbased surveys. Antitransits and transits will both provide valuable insight into the end states of common envelope evolution and of stable mass transfer, resolving issues that must be understood before we can fully unravel the progenitor puzzle.
\end{abstract}

Keywords. supernovae - gravitational lensing

Lensing and Type Ia Progenitors. When a WD orbits a non-degenerate star, the orbital inclination may allow the WD to transit its companion; the probability is proportional to the ratio of the companion's radius and the orbital separation. Transits contain information about the WD and the binary orbit. The small size of the WD means, however, that exquisitely sensitive measurements are required, like those currently being conducted by the Kepler space mission, designed to discover transits of Sun-like stars by Earth-like planets. Kepler has indeed discovered transiting WDs (Rowe et al. 2010). Population synthesis calculations indicate that Kepler should discover dozens of additional transiting systems (Di Stefano 2011). Each provides information about the masses of the components and their orbit. The result is that we are developing a remarkable set of data telling us about an important intermediate state: the state that bridges the gap between primordial binaries and binaries in which mass is transferred onto a WD, producing cataclysmic variables, novae, symbiotic binaries, accretion-induced collapses and, in some cases, SNe Ia.

Perhaps even more remarkable is that, if the WD is far enough from the companion, the effect that will dominate is not the blocking of light from the companion, but is instead gravitational lensing, in which the compact WD is able to deflect light from the companion, so that we actually see an antitransit. When the WD serves as a lens, we are able to directly measure its gravitational mass.

Figure 1 shows which types of possible SNe Ia progenitors are likely to show antitransits, as opposed to transits. Figure 2 shows antitransit profiles expected for some binaries that happen to be end states of a first phase of stable mass transfer; binaries in the top two panels could correspond to SNe Ia progenitors. For more details, see Di Stefano 2010, 2012 . 


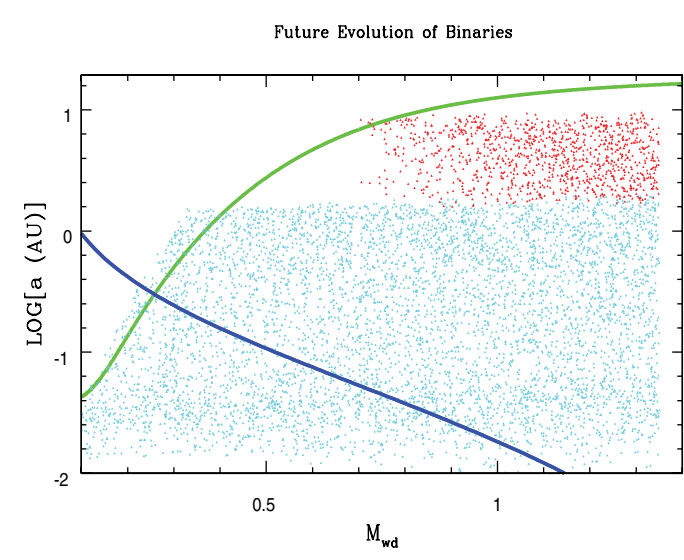

Figure 1. $\log _{10}(a)$ versus $M_{W D}$ : Systems in the region above (below) the blue [darker] curve will exhibit antitransits (transits) The green [lighter] curve shows the end state of stable mass transfer. Points in red [darker] will become symbiotic binaries in which the there is a large enough reservoir of mass that, should the WD capture and retain $10 \%$ of the winds leaving the giant, it will reach a mass at least equal to $M_{C}$. Points in cyan [lighter] could become nuclear-burning WDs; the mass reservoir in the systems shown is large enough that the WD will achieve at least $M_{C}$ if it can retain $60 \%$ of the mass lost by the donor.

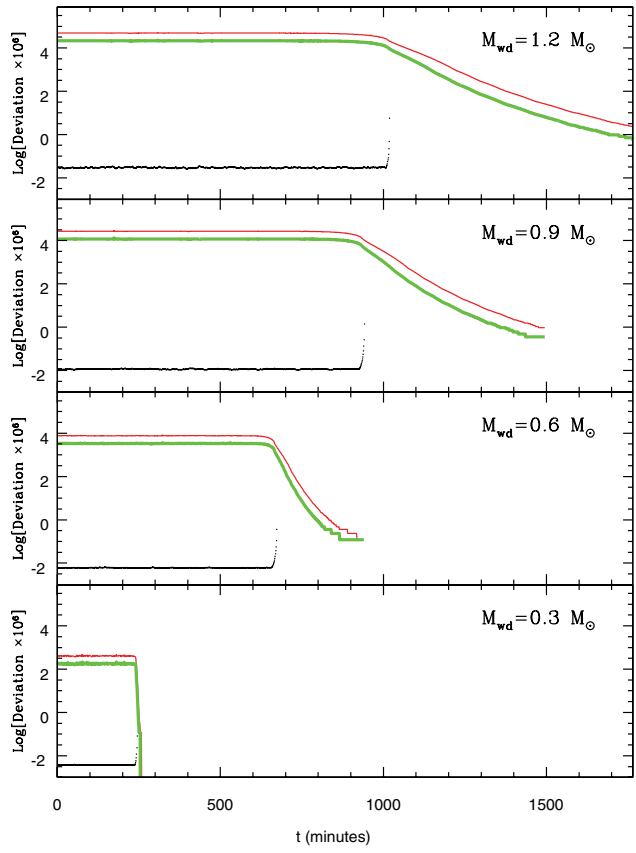

Figure 2. Transit events by WDs that emerge from stable mass transfer. The period/core-mass relationship (Rappaport et al. 1995) applies, so that more massive WDs are in larger orbits. Shown here is the deviation, starting at the center of the transit at $t=0$, in PPM versus time. The WD is positioned at the center of the star it transits at $t=0$. Black [lower] curves show what the transit signature would be if the WD had no mass. Red [upper] shows the signature for a pointlike lens with the WD's mass; this is a pure lensing. Green [intermediate] shows the true light curve, including the finite-lens size and gravitational lensing. Note the large size of the lensing effect.

\section{Acknowledgements}

This work was supported in part by NSF under AST-0908878.

\section{References}

Di Stefano, R. 2011, AJ, 141, 142

Di Stefano, R. 2010, American Institute of Physics Conference Series, 1314, 196

Di Stefano, R. 2012, in preparation

Farmer, A. J. \& Agol, E. 2003, ApJ, 592, 1151

Rappaport, S., et al. 1995, MNRAS, 273, 731

Rowe, J. F., et al. 2010, ApJL, 713, L150

Sahu, K. C. \& Gilliland, R. L. 2003, ApJ, 584, 1042

van Kerkwijk et al. 2010, ApJ, 715, 51 